\title{
Effects of Metoclopramide Orally Disintegrating Tablet in Optimizing Late Outpatient Endoscopy: A Randomized Double-Blind Placebo-Controlled Clinical Trial
}

\author{
Patrick P. Basu',2,3, Niraj J. Shah4, Hemanth Hampole³, Nitya Krishnaswamy3, \\ Mark M. Aloysius ${ }^{2 *}$ \\ ${ }^{1}$ A Columbia University College of Physicians \& Surgeons, New York, USA \\ ${ }^{2}$ Kings County Hospital, Internal Medicine, New York, USA \\ ${ }^{3}$ Forest Hills Hospital, Forest Hills, New York, USA \\ ${ }^{4}$ James Peters VA Medical Center, New York, USA \\ Email: ${ }^{*}$ mark.aloysius5@gmail.com
}

Received 2 March 2014; revised 3 April 2014; accepted 11 April 2014

Copyright (C) 2014 by authors and Scientific Research Publishing Inc.

This work is licensed under the Creative Commons Attribution International License (CC BY).

http://creativecommons.org/licenses/by/4.0/

c) (i) Open Access

\begin{abstract}
Aims: Metoclopramide has been used as a gastro-kinetic agent but the safety and effects of different preparations of metoclopramide on preparation for outpatient endoscopy are largely unknown. The study aimed to evaluate the (1) Safety of using ODT (orally disintegrating tablet) or TT (traditional tablet) metoclopramide pre-endoscopy in non-fasting patients compared with placebo (2) Impact of ODT or TT metoclopramide on sedation and recovery times compared with placebo. Methods: A double blind randomized placebo-controlled trial with 3 arms; Metoclopramide ODT $(n=43)$, Metoclopramide TT $(n=43)$ and Placebo $(n=43)$. Results: Metoclopramide ODT had fewer adverse events compared with TT or placebo. Recovery time was significantly shorter with use of either metoclopramide versus placebo $(P<0.001) \&$ total sedation time was also significantly shorter in the metoclopramide groups versus placebo $(P<\mathbf{0 . 0 0 1})$. Conclusion: Metoclopramide ODT is safe and beneficial in endoscopic procedures requiring limited access to food and liquid.
\end{abstract}

\section{Keywords}

Metoclopramide; ODT; Endoscopy

\footnotetext{
*Corresponding author.
}

How to cite this paper: Basu, P.P., et al. (2014) Effects of Metoclopramide Orally Disintegrating Tablet in Optimizing Late Outpatient Endoscopy: A Randomized Double-Blind Placebo-Controlled Clinical Trial. Open Journal of Gastroenterology, 4, 187-191. http://dx.doi.org/10.4236/ojgas.2014.44028 


\section{Introduction}

Endoscopy is a common procedure that allows minimally invasive observation and biopsy of mucosal surfaces of the gastrointestinal (GI) tract [1].

Endoscopy is performed using moderate sedation without hospitalization [2] but anesthesiologist guidelines prohibit the consumption of liquid for within 2 hours of the procedure.

Use of prokinetic agents (e.g., erythromycin, metoclopramide) before endoscopy may allow better visualization of the GI tract [3].

Metoclopramide is a central and peripheral nervous system dopamine antagonist with prokinetic and antiemetic properties [4].

- Peak plasma concentration of oral metoclopramide occurs <2 hours after administration [4] [5].

- Orally disintegrating agents have a much faster peak plasma concentration with prompt delivery of action within 30 minutes.

- Time of drug disintegration orally is approximately $60 \mathrm{sec}$.

Central nervous system action of metoclopramide may impact sedation and recovery of patients when used with some sedatives (e.g., propofol) [2].

An orally disintegrating tablet formulation of metoclopramide that is bioequivalent to traditional metoclopramide tablets has been developed to be taken without liquid [5].

This formulation would allow administration of metoclopramide $<2$ hours before an endoscopic procedure and may improve visibility by allowing performance of the procedure during the time of peak plasma drug concentration.

\section{Objectives}

- To evaluate the safety of metoclopramide ODT during outpatient endoscopic procedures

- To evaluate the effect of ODT compared with TT or placebo on the endoscopic total sedation and recovery times

\section{Methods}

\subsection{Patient Population}

\subsubsection{Eligibility Criteria}

Patients requiring elective EGD (esophagogastroduodenoscopy)

Benign disorders of the upper GI (gastrointestinal) tract

Non-diabetic

Npn-obese

\subsubsection{Exclusion Criteria \\ Active GI bleeding \\ Diabetes mellitus \\ Neuromuscular diseases \\ Gastric motility disorders \\ Gastric malignancy \\ Use of GI motility-altering drugs \\ Body mass index $>33 \mathrm{~kg} / \mathrm{m}^{2}$}

\subsection{Study Location}

Patient recruitment, study and data collection was carried out at an outpatient endoscopic facility in NY.

\subsection{Study Design}

The study design was a double blind randomized placebo-controlled trial with 3 treatment arms (Placebo, Metoclopramide ODT and Metoclopramide TT). Sequential randomization was carried out using envelopes.

Patients received a solid meal (i.e., approximately $28.3 \mathrm{~g}$ of chicken, turkey, or tuna with $14.1 \mathrm{~g}$ of potato or 
fruit salad without mayonnaise or diary products, and $354.9 \mathrm{~mL}$ of water) 5 hours before their endoscopic procedure.

Patients were randomized to receive either placebo, metoclopramide traditional tablet (TT) $10 \mathrm{mg} 30$ minutes before endoscopy, or metoclopramide ODT $10 \mathrm{mg} 20$ minutes before endoscopy

VIT B12 was chosen as placebo because it has a distant taste like ODT tab on the tongue which also has a distinct taste. No liquids were allowed except for the patients' own saliva.

\subsection{Procedure}

Patients scheduled to receive late afternoon outpatient pan endoscopy for the obtainment of 10 biopsies were included (Duodenum-2, Antro-gastric body-4, cardia-1, fundus-1, esophagus-2).

Propofol $7 \mu \mathrm{g} / \mathrm{kg} / \mathrm{min}$, was administered intravenously by an anesthesiologist before endoscopy

The depth of sedation was conscious sedation ASA II. Patient was considered sedated when there was no agitation/resistance to the procedure and was considered non-sedated when able to perform a head raise test on command.

\subsection{Outcomes}

This was a safety and efficacy pilot study and the following outcomes were measured

1) Safety of metoclopramide (ODT, TT) vs placebo in late outpatient endoscopy

2) The effect of metoclopramide (ODT, TT) vs placebo on total sedation and recovery time

\subsection{Statistics}

Mean dosage of Propofol was $65 \mathrm{mg}$ in ODT, $75 \mathrm{mg}$ in Oral Metoclopramide and about $100 \mathrm{mg}$ in Placebo with a mean BMI of all groups 27.8. Total sedation time and recovery time were ascertained and comparisons among the treatment groups were performed with one-way ANOVA. Adverse events were monitored throughout the study.

\section{Results}

Patient demographics (age ethnicity and BMI) between study groups were similar. All patients recruited complied with instructions and there were no drop out from the study (Table 1).

Study results demonstrated significantly reduced sedation times for both TT and ODT preparations of metoclopramide when compared with placebo but these times did not differ significantly between TT and ODT (Figure 1). A similar trend was observed in recovery times for patients (Figure 2).

Tolerability was significantly better with metoclopramide ODT preparation compared with metoclopramide TT preparation or placebo. Metoclopramide ODT group did not experience any side events but metoclopramide TT and placebo groups experienced dizziness (ODT group 46\%, placebo group 28\%) and confusion (ODT group 26\%, placebo group 16\%) respectively (Table 2).

\section{Table 1. Patient demographics.}

\begin{tabular}{lccc}
\hline \multicolumn{1}{c}{ Parameter } & $\begin{array}{c}\text { Placebo } \\
(\mathbf{n}=\mathbf{4 3})\end{array}$ & $\begin{array}{c}\text { Metoclopramide tablet } \\
(\mathbf{n}=\mathbf{4 3})\end{array}$ & $\begin{array}{c}\text { Metoclopramide ODT } \\
(\mathbf{n}=\mathbf{4 3})\end{array}$ \\
\hline Mean age (range), & 45 & 46 & 48 \\
Male:Female, $\mathrm{n}$ & $2: 1$ & $2: 2$ & $2: 1$ \\
Race, n (\%) & & & 13 \\
White & 13 & 16 & $\mathbf{1 2}$ \\
Black & 11 & 10 & 9 \\
Hispanic & 10 & 5 & 9 \\
Asian & 9 & 28.3 & 28 \\
Body weight, n (range) BMI & 27.4 & & \\
\hline
\end{tabular}

ODT: orally disintegrating tablet. 
Table 2. Adverse events reported during endoscopy and patient recovery.

\begin{tabular}{cccc}
\hline Parameter & $\begin{array}{c}\text { Placebo } \\
(\mathbf{n}=\mathbf{4 3})\end{array}$ & $\begin{array}{c}\text { Metoclopramide TT } \\
(\mathbf{n}=\mathbf{4 3})\end{array}$ & $\begin{array}{c}\text { Metoclopramide ODT } \\
(\mathbf{n}=\mathbf{4 3})\end{array}$ \\
\hline Dizziness, $\mathrm{n}(\%)$ & $20(46)$ & $11(26)$ & $0(0)$ \\
Confusion, n (\%) & $12(28)$ & $7(16)$ & $0(0)$ \\
\hline
\end{tabular}

ODT: orally disintegrating tablet; TT: traditional tablet.

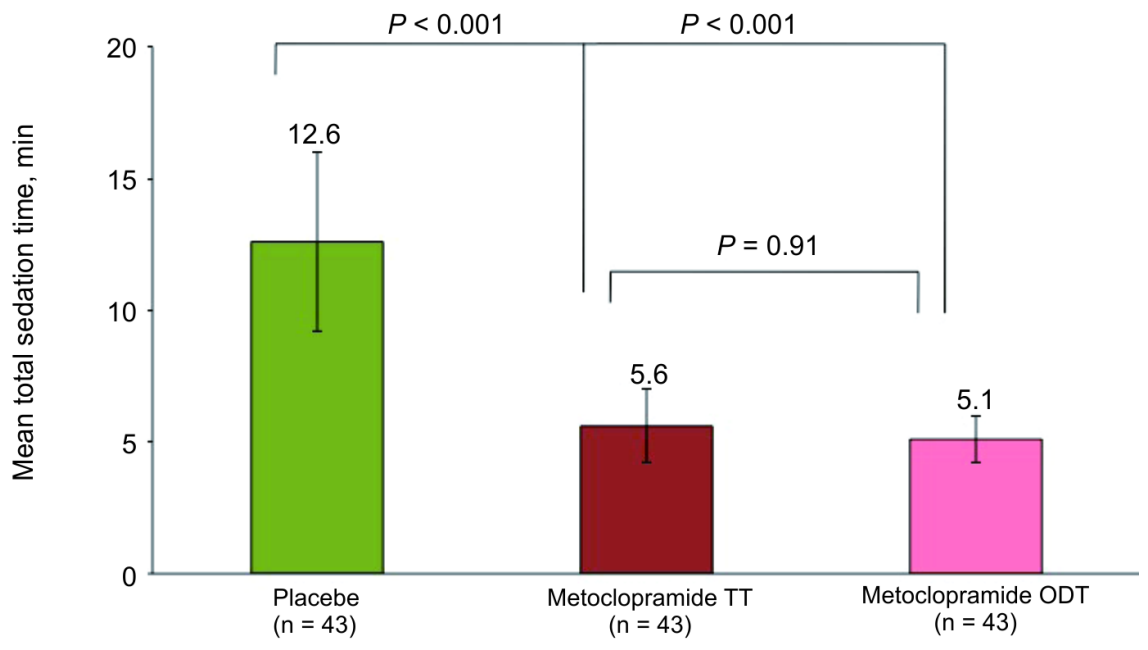

Figure 1. Total sedation time in patients who received metoclopramide before endoscopy. Patients received placebo, metoclopramide TT $10 \mathrm{mg} 30$ minutes before endoscopy, or metoclopramide ODT $10 \mathrm{mg} 20 \mathrm{~min}$ before endoscopy. Propofol was used to achieve moderate sedation for the procedure. Error bars represent standard deviation. ODT, orally disintegrating tablet; TT, traditional tablet. Recovery time was significantly shorter with use of either metoclopramide medication versus placebo $(P<0.001)$. There was no difference in recovery time between the different formulations of metoclopramide $(P=0.75$; Figure 2$)$.

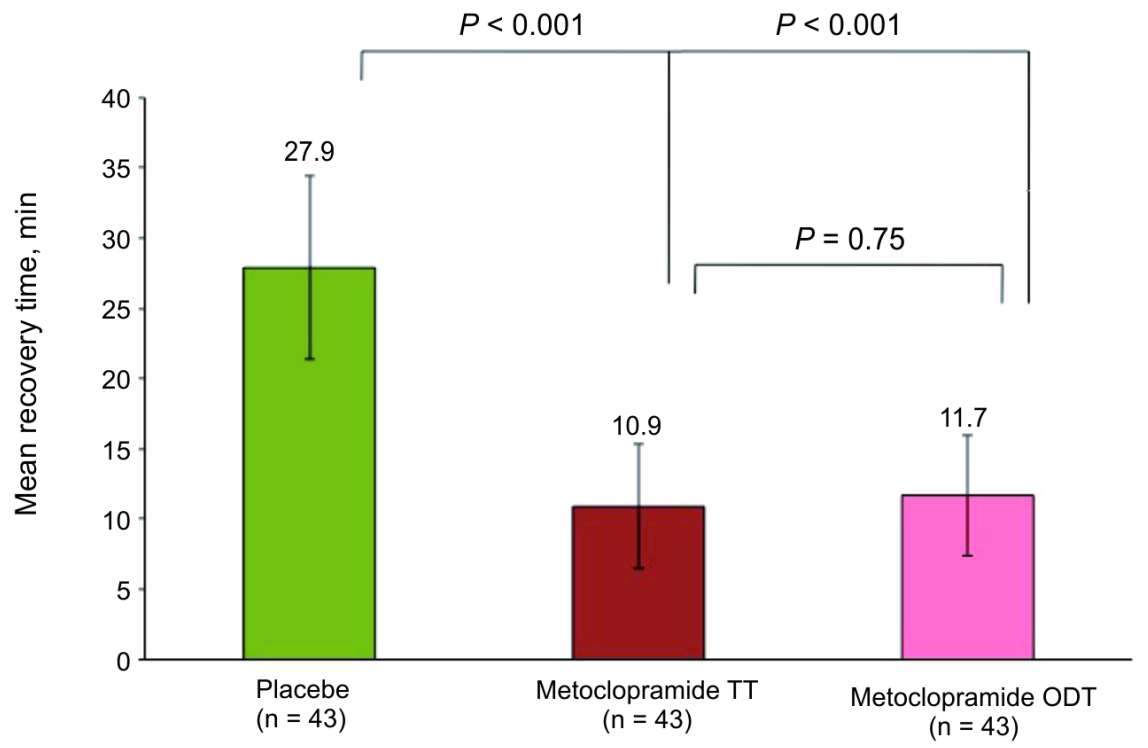

Figure 2. Total recovery time after sedation with propofol for endoscopy. Patients received placebo, metoclopramide TT $10 \mathrm{mg} 30$ minutes before endoscopy, or metoclopramide ODT 10 mg 20 min before endoscopy. Error bars represent standard deviation. ODT, orally disintegrating tablet; TT, traditional tablet. Major adverse events were less prevalent in the metoclopramide ODT group versus placebo or metoclopramide TT groups (Table 2). 


\section{Discussion}

Metoclopramide promotes gastric emptying based upon its ability to be an agonist of motilin receptors. The usefulness of metoclopramide as a prokinetic agent in upper gastro-intestinal endoscopy for bleeding emergencies iswell documented for its role in optimal visualization and reduced rates of second look endoscopy [6]. Metoclopramide has also been studied quite extensively in capsule endoscopy on its completion and yield and has been found to increase completion but not yield.

However, the role of metoclopramide in outpatient endoscopy, its impact on sedative dose, total sedation duration and recovery time have not been studied before in humans, although a study using pre-EGD metoclopramide has been shown to be counterproductive in canines [7]. The oldest known and the only related human study to date, has been from France where Metoclopramide was used IV before gastroscopy in patients over 75 years of age and was found to alleviate nausea and anxiety. However, no effect on sedation recovery times or safety was documented in this study [8].

Metoclopramide reduced total sedation and recovery time required for outpatient endoscopy versus placebo. This reduction in time may relate to greater endoscopic visibility facilitated by metoclopramide administration. Metoclopramide ODT was equivalent to a traditional tablet formulation of metoclopramide and elicited fewer adverse events than both placebo or metoclopramide TT, suggesting that metoclopramide ODT may be beneficial in endoscopic procedures requiring limited access to food and liquid

We demonstrate the usefulness of metoclopramide preparations (TT and ODT) to significantly reduce the total endoscopy duration as a direct consequence of enhanced visibility achieved by better gastric emptying and therefore resulting in reduced requirement for sedative and reducing recovery times.

To the best of our knowledge, this is the first time in scientific literature a direct comparison of clinical effects has been made through a randomized controlled trial by using metoclopramide preparations ODT vs.TT before outpatient upper GI endoscopy.

\section{Conclusion}

We conclude that ODT preparation is better than the TT preparation in rendering similar benefit without the burden of intolerance. We recommend routine use of ODT metoclopramide in patients undergoing routine upper gastrointestinal endoscopy.

\section{References}

[1] Minoli, G., Meucci, G., Bortoli, A., Garripoli, A., Gullotta, R., Leo, P., Pera, A., Prada, A., Rocca, F. and Zambelli, A. (2000) The ASGE Guidelines for the Appropriate Use of Colonoscopy in an Open Access System. Gastrointest Endosc, 52, 39-44.

[2] Cohen, L.B., Delegge, M.H., Aisenberg, J., Brill, J.V., Inadomi, J.M., Kochman, M.L., et al. (2007) AGA Institute Review of Endoscopic Sedation. Gastroenterology, 133, 675-701.

[3] Frossard, J.L., Spahr, L., Queneau, P.E., Giostra, E., Burckhardt, B., Ory, G., et al. (2002) Erythromycin Intravenous Bolus Infusion in Acute Upper Gastrointestinal Bleeding: A Randomized, Controlled, Double-Blind Trial. Gastroenterology, 123, 17-23.

[4] Albibi, R. and McCallum, R.W. (1983) Metoclopramide: Pharmacology and Clinical Application. Annals of Internal Medicine, 98, 86-95.

[5] Fass, R., Pieniaszek, H.J. and Thompson, J.R. (2009) Pharmacokinetic Comparison of Orally-Disintegrating Metoclopramide with Conventional Metoclopramide Tablet Formulation in Healthy Volunteers. Alimentary Pharmacology \& Therapeutics, 30, 301-306.

[6] Barkun, A.N., Bardou, M., Martel, M., Gralnek, I.M. and Sung, J.J. (2010) Prokinetics in Acute Upper GI Bleeding: A Meta-Analysis. Gastrointestinal Endoscopy, 72, 1138-1145.

[7] Monroe, W.E., Leib, M.S., Matz, M.E., Davenport, D.J., Nelson, L.P. and Kenny, J.E. (1992) Evaluation of Metoclopramide Hydrochloride as an Aid for Passage of a Flexible Endoscope into the Duodenum of Dogs. American Journal of Veterinary Research, 53, 149-152.

[8] Korn, M. (1984) Use of Metoclopramide in the Preparation of Patients over 75 for Gastroscopy. The Annals of Gastroenterology \& Hepatology (Paris), 20, 225-227. 